Etnográfica

Revista do Centro em Rede de Investigação em

Antropologia

vol. 21 (3) | 2017

Vol. 21 (3)

\title{
Trata de personas, tráfico de migrantes y la gobernabilidad de la migración a través del crimen
}

Human trafficking, human smuggling and migration governability through crime

\section{Guilherme Mansur Dias}

\section{CpenEdition}

Journals

Edición electrónica

URL: https://journals.openedition.org/etnografica/5026

DOI: 10.4000/etnografica. 5026

ISSN: 2182-2891

\section{Editor}

Centro em Rede de Investigação em Antropologia

Edición impresa

Fecha de publicación: 1 octubre 2017

Paginación: 541-554

ISSN: 0873-6561

\section{Referencia electrónica}

Guilherme Mansur Dias, «Trata de personas, tráfıco de migrantes y la gobernabilidad de la migración a través del crimen», Etnográfica [En línea], vol. 21 (3) | 2017, Publicado el 24 marzo 2018, consultado el 09 febrero 2022. URL: http://journals.openedition.org/etnografica/5026 ; DOI: https://doi.org/10.4000/ etnografica. 5026

\section{(c) (i) (9)}

Etnográfica is licensed under a Creative Commons Attribution-NonCommercial 4.0 International License. 


\section{Trata de personas, tráfico de migrantes y la gobernabilidad de la migración a través del crimen}

\section{Guilherme Mansur Dias}

El presente artículo reflexiona sobre el rol de los conceptos de "trata de personas" y "tráfico ilícito de migrantes" en la dinámica de gobernabilidad de la migración internacional contemporánea. Para ello, se realiza un análisis crítico de la difusión de estos temas en el derecho internacional, basado en trabajo anterior que investigó la relación entre los campos migratorio y de seguridad, y la reproducción de las políticas internacionales anti-trata/anti-tráfico. La investigación etnográfica se llevó a cabo entre los años 2011 e 2013 en la ciudad de Viena, con un amplio análisis de las prácticas de organizaciones internacionales que trabajan en los campos de la migración y la justicia penal. El artículo también explora la cuestión de la "víctima" y la "victimización" con base en la descripción de un movimiento de refugiados afectado por las retóricas y leyes de lucha contra el tráfico.

PALABRAS-CLAVE: trata de personas, tráfico ilícito de migrantes, gobernabilidad migratoria, refugiados, Viena.

Human trafficking, human smuggling and migration governability through crime - This article examines the role played by the concepts of "human trafficking" and "human smuggling" in the dynamics of contemporary migration governability. It does so through a critical analysis regarding the diffusion of these topics in international law. The conclusions are based on earlier work addressing the relationship between the fields of migration and security/crime, and the reproduction of international anti-trafficking policies and legislation. The ethnographic research was conducted from 2011 to 2013 in the city of Vienna, focusing on a comprehensive analysis of the practices of international organizations that deal with migration and criminal justice. The article also explores the issue of "victim" and "victimization", taking into account the description of a movement of refugees affected by the rhetoric and laws against trafficking.

KEYWORDS: human trafficking, human smuggling, migration governability, refugees, Vienna.

DIAS, Guilherme Mansur (guiboamansur@yahoo.com.br) - Programa de Pós-Graduação em Sociologia e Antropologia - Universidade Federal do Rio de Janeiro (PPGSA/UFRJ), Brasil; Centre de Recherche Sociologique sur le Droit et les Instituitions Pénales (CESDIP), Francia. 


\section{LA TRATA DE PERSONAS Y EL TRÁFICO ILÍCITO DE MIGRANTES}

Entre los conceptos relacionados con la movilidad humana que han adquirido más importancia en los últimos años, la "trata de personas" y el "tráfico ilícito de migrantes" han obtenido gran protagonismo. ${ }^{1}$ En las últimas dos décadas, estos conceptos se han convertido en objeto privilegiado de la agenda política internacional, con la participación de actores como el Departamento de Estado y el Congreso de Estados Unidos, la Unión Europea y las Naciones Unidas (Wong 2005).

Una de las razones de la centralidad de estos temas se relaciona con la firma y ratificación de la Convención de las Naciones Unidas contra la Delincuencia Organizada Transnacional, en 2000 (UNODC 2004). Con sus protocolos de tráfico ilícito de migrantes y de trata de personas, esta convención marca una transposición de temas antes tratados esencialmente en las convenciones internacionales de derechos humanos y trabajo a la estructura criminal de la ONU.

Por otra parte, la inclusión de la cuestión de la movilidad humana en los debates internacionales sobre la delincuencia refleja el enfoque de las formas de conocimiento históricamente asociadas con el fenómeno penal - derecho penal, narrativa popular penal y criminología - como una herramienta de gobierno de temas sociales (Simon 2007). Por lo tanto, aunque se presenta bajo el ropaje de los derechos humanos y la protección de las víctimas, los protocolos de tráfico ilícito de migrantes y la trata de personas reiteran el vínculo entre la migración y la seguridad, reforzando prácticas de criminalización y el control migratorio.

En el caso de la trata de personas, a pesar de que los debates en torno al tema se remontan a la década de 1980 y a la preocupación emergente de los grupos de mujeres con la relación entre la trata y el turismo sexual en el sudeste asiático (Piscitelli 2011), el vínculo que la trata establece con el tema de la migración y la seguridad es notorio. Importantes estudios han cuestionado la intersección entre la trata de personas y el control migratorio, señalando las consecuencias negativas de la difusión de este concepto en diferentes contextos contemporáneos.

I Este artículo es resultado de mi investigación de doctorado en el Departamento de Antropología de la Universidad Estatal de Campinas (Unicamp) entre los años 2010 y 2014 (Dias 2014a). Algunas reflexiones de este texto se discutieron en dos presentaciones en la Faculdad Lationamericana de Ciencias Sociales (FLACSO): la reunión del grupo de trabajo "Migración, Cultura y Políticas" (Clacso), celebrada en Quito (16 a 18 de marzo de 2015) y en la mesa redonda "Políticas Públicas en Torno a la Trata de Personas y el Tráfico de Migantes: Desafíos para su Formulación e Implementación”, que se realizó en Quito, el 28 de mayo de 2015. Agradezco los comentarios de los compañeros del grupo, especialmente Gioconda Herrera, debido a sus preguntas sobre el tema de la victimización. Además, mi agradecimiento a Silvia Zelaya por revisar el texto en español. 
Las etnografías e investigaciones realizadas en países como Nepal, Indonesia, Alemania, Francia, Italia, España, Brasil, Emiratos Árabes Unidos, Canadá y Estados Unidos muestran que la difusión de las políticas anti-trata causan la restricción y la criminalización de la movilidad de ciertos grupos e individuos, como personas involucradas en el comercio sexual e inmigrantes indocumentados (Augustin 2007; Bernstein 2012; Dias y Sprandel 201 1; Doezema 2005, 2010; Lee 201 1; Mahdavi 201 1; Kempadoo 2005; Olívar 2016; Piscitelli 2008, 2011 ; Silva y Blanchette 2010; Sprandel y Dias 2010; Teixeira 2008; Ticktin 2008).

Elizabeth Bernstein (2012), feminista estadounidense estudiosa del tema, describe como las políticas de "trata de personas" han permitido el avance de sectores evangélicos en la agenda de los derechos humanos a nivel internacional. ${ }^{2}$ De acuerdo con la autora, los evangélicos han optado por renunciar a discusiones aislacionistas como el aborto y el matrimonio gay para difundir una teología de la justicia social orientada a la escena internacional (Bernstein 2012: 25 1). La adopción de la agenda de lucha contra la trata puede ser vista, por lo tanto, como el resultado de una estrategia política exitosa acerca de un tema "consensual" para reforzar los ideales políticos en torno al género y la sexualidad que reafirman suposiciones acerca de la supremacía moral del matrimonio (Bernstein 2012: 250).

En este proceso, las campañas anti-trata se multiplicaron, basándose en "fantasías de violencia en el primer mundo" e individualizando/personalizando los problemas sociales y estructurales inherentes al capitalismo, además de condenando a las empresas "malas" y los gobiernos que se oponen a la hegemonía occidental (Kempadoo 2016). Del mismo modo, la categoría "trata de personas" ocupa un lugar importante en el discurso político difundido por organizaciones internacionales que trabajan con el tema de la migración/seguridad y que concatena acciones simultáneas de control de la movilidad con cierta retórica de los derechos humanos, basado en las ideas de "víctima", "vulnerabilidad" y "violación de los derechos" (Clavijo y Magliano 2013; Dias 2014a). ${ }^{3}$

En el caso del tema "tráfico ilícito de migrantes", a su vez, la presión para la inclusión de un protocolo sobre el tema en el ámbito de una Convención Penal se debió a los esfuerzos de los gobiernos de Austria e Italia, y obtuve pleno apoyo de otros países europeos y de los Estados Unidos. En el momento de la negociación de la Convención - finales de 1990 -, la llegada de inmigrantes a Europa por el Mar Mediterráneo fue un tema destacado en la política europea, como lo sigue siendo en la actualidad.

Los diversos cambios vividos en Europa - el colapso del socialismo y las diferentes redefiniciones territoriales, económicas y políticas después de la Guerra

2 Mi agradecimiento a Adriana Piscitelli por la indicación del trabajo de Elizabeth Bernstein.

3 Para un análisis más detallado del papel de estas organizaciones en la reproducción de mecanismos contemporáneos de gobernanza migratoria vinculados al control poblacional, ver Dias (2014b). 
Fría - llevaron a un fuerte resurgimiento del discurso conservador, ahora comprometido para contrarrestar la presencia de inmigrantes. En este discurso, se estimuló la asociación de los extranjeros con el "aumento de la delincuencia", lo que dice más sobre el pánico asociado a reacciones anti-inmigrantes que sobre una vinculación necesaria entre el aumento correlativo de fenómenos como la migración y la delincuencia.

En ese período, Austria estaba recibiendo una afluencia considerable de personas de Europa del Este debido a la caída del régimen socialista, además del aumento de las solicitudes de asilo para las personas huyendo del conflicto en la antigua Yugoslavia. Por su parte, Italia estaba recibiendo un número significativo de personas en Lampedusa y otras regiones de la costa del país. Además de las corrientes procedentes de África, el gobierno italiano estaba preocupado por las corrientes de kurdos, paquistaníes, iraquíes y chinos, así como por la migración de los albaneses por el Mar Adriático, debido a los conflictos en los Balcanes.

Las autoridades austríacas e italianas, por lo tanto, tuvieron un amplio apoyo para instalar el tema de la migración en la estructura institucional de las Naciones Unidas encargada de tratar con el control/represión de drogas y el crimen. Desafortunadamente, en las negociaciones del "Protocolo contra el Tráfico Ilícito de Migrantes por Tierra, Mar y Aire” (UNODC 2004), las extensas discusiones referidas a la realidad de la migración de grupos e individuos se convirtieron en un debate polarizado entre "soberanía nacional" y, en oposición, "los derechos fundamentales de los migrantes".

Así, la cuestión de la migración transfronteriza quedó interpretada sistemáticamente en una óptica penal y por medio de conceptos tales como "coyotes", "grupos criminales", "contrabando" y "violación de las fronteras", haciendo conectar aún más la movilidad humana con las nociones de delincuencia y seguridad. La fundamentación del "Protocolo contra el Tráfico Ilícito de Migrantes” está basada en una perspectiva binaria donde una tajante línea marca una distinción entre actividades legales e ilegales; entre traficante y migrante; entre trata y tráfico (Velasco 2015).

En resumen, cuando se habla de la importancia adquirida por los temas de la trata de personas/tráfico de migrantes a nivel internacional, hay que tener en cuenta las políticas de seguridad de los países hegemónicos y, con ellas, la exportación de modelos de gobernabilidad migratoria que simplifican la realidad, además de transformar a los individuos en víctimas pasivas e insistentemente vulnerables.

\section{VÍCTIMAS Y VICTIMIZACIÓN}

Los autores que reflexionan sobre los campos humanitarios - especialmente sobre los campamentos de refugiados - han puesto de relieve la aparición de dispositivos "humanitarios" de la policía y fuerzas militares en el control de 
las poblaciones estigmatizadas (Agier 2006; Agamben 2004). Estos estudios reiteran la fuerza del discurso en torno a las "víctimas absolutas", las masas de las personas despojadas de toda sociabilidad y tratados como "víctimas puras", que justificarían la acción misma de las organizaciones no gubernamentales y organizaciones internacionales especializadas.

El trabajo de Didier Fassin $(2001$, 2006), a su vez, expande estas ideas, mostrando como la invención del "sufrimiento social" y la difusión de perspectivas morales vinculadas con el sufrimiento se relacionan con las prácticas políticas en el tratamiento de las poblaciones y grupos subalternizados. El fortalecimiento del sentimiento de compasión en este caso coincide con la creación de cuerpos dóciles y sumisos, principales objetivos de la intervención humanitaria.

Estas reflexiones nos interesan, ya que ayudan a pensar en la transferencia del significado de "víctima criminal" a la "víctima humanitaria". La confluencia del discurso humanitario y de la justicia penal en los últimos años llevó a una introducción vigorosa de los conceptos de "víctima" y "vulnerabilidad" en los debates sobre migración y la consiguiente simplificación de la experiencia migratoria. Tal cambio interpretativo genera la comprensión de las trayectorias de migración no convencionales como "tráfico" o "trata" de personas. ${ }^{4}$

En el caso de la trata/tráfico ilícito de migrantes, la confluencia de las intervenciones y discursos humanitarios y criminales hace que el tema de la "víctima" se vuelva aún más complejo y delicado. Como muestran Piscitelli y Lowenkron (2015) en sus reflexiones sobre los regímenes discursivos y legales sobre la trata de personas en Brasil y España, para acceder a algún tipo de reconocimiento, las personas "víctimas de la trata" deben ser reconocidas como "víctimas reales" por los Estados, mientras que, paradójicamente, en la práctica, los reconocidos como "víctimas reales" son a menudo tratados como "inmigrantes ilegales" a ser deportados o incluso criminalizados.

Así pues, si en España la ampliación del debate sobre la trata de personas como un problema social ha utilizado la retórica de los derechos humanos, con base en una figura de víctima utilizada en las campañas generalizadas para "sensibilizar" a la población, las personas detenidas en las inspecciones de policía no pueden acceder a la condición jurídica de las víctimas de este delito, a menos que decidan convertirse en "testigos" o "denunciantes" en casos penales (Piscitelli y Lowenkron 2015).

La complejidad adquirida por las consecuencias de las políticas contemporáneas contra la trata es evidente cuando la victimización se convierte en una herramienta de intervención que a menudo viola los derechos humanos,

4 Para la comprensión de como el uso de categorías como prostitución, turismo sexual y tráfico, por parte de representantes de gobiernos, proyectos sociales y académicos, borra la diversidad entre las situaciones e impulsos de la gente que viaja, participa en redes que facilitan los viajes y vive del comercio sexual, ver Augustin (2005). 
mientras que al mismo tiempo es la única alternativa para que algunos migrantes reclamen derechos de los Estados-nación. Durante las negociaciones del "Protocolo contra el Tráfico Ilícito de Migrantes", por ejemplo, la delegación filipina intentó incluir un párrafo que hace referencia precisamente a los derechos de los migrantes y su condición de víctimas, con el fin de contrarrestar las proposiciones de restricción de la migración por las delegaciones austriaca e italiana (Dias 2014a: 65). ${ }^{5}$

En los debates sobre la trata de personas, la noción de víctima se incrementa también por la idea de la vulnerabilidad, especialmente relacionada con las mujeres y los niños. Este concepto se destaca por ser incluido en el preámbulo y la definición de trata del Protocolo de Palermo. Según Ela Castilho (2013), la incorporación de la idea de la vulnerabilidad en el Protocolo de Palermo sigue la tendencia de la inclusión del término en otros documentos internacionales, empezando por la Declaración de Viena y el Programa de Acción adoptado por la Conferencia Mundial de Derechos Humanos en 1993 (ACNUDH 2013).

Sin embargo, a diferencia de lo que ocurre (donde los grupos vulnerables y los factores de riesgo indicados son expresamente limitados), documentos dirigidos a la defensa de los derechos humanos se han encargado de dejar explícito que no hay personas vulnerables por sí mismas, sino que alguien se convierte en vulnerable en ciertos contextos o como resultado de ciertas prácticas o situaciones. ${ }^{6} \mathrm{O}$ sea, es esencial que la vulnerabilidad se entienda como categoría relacional, en el propósito de garantizar la protección a ciertas personas y grupos sociales.

De todos modos, es importante reflexionar con más calma sobre las implicaciones del proceso de cambio conceptual y político que ha dado lugar a la transformación de los agentes sociales en "víctimas". La transformación interpretativa, que culminó con la individualización y focalización de problemas sociales, apela a la aparición cada vez más contundente de la "víctima" en la escena política, lo que coincide con la difusión de las "políticas de reparación" a nivel internacional. ${ }^{7}$

5 Sobre las contradicciones inherentes a la construcción del Protocolo de Palermo, ver Doezema (2005).

6 El artículo 24 de la Declaración y Programa de Acción de Viena de 1993 establece que "Debe darse gran importancia a la promoción y protección de los derechos humanos de las personas pertenecientes a grupos que han pasado a ser vulnerables, en particular los trabajadores migratorios, a la eliminación de todas las formas de discriminación contra ellos y al fortalecimiento y la aplicación más eficaz de los instrumentos de derechos humanos" (ACNUDH 2013: \$24).

7 La obra de Marcia Sprandel (2004) discute este tema de una manera muy singular, destacando el papel de los bancos multilaterales de desarrollo y organizaciones internacionales en este proceso de transformación de los actores sociales en "víctimas". La autora describe cómo desde la década de 1990 la orientación de las políticas para hacer frente a la pobreza se basó en un cálculo matemático (IDH), que supone un "pobre" individualizado, pero universal y genérico, carente de historias de vida y narrativas. En Brasil, estas acciones no sólo tuvieron malos resultados, así como debilitaran los movimientos sociales organizados (Sprandel 2004). 
Al reflexionar sobre la difusión de estas políticas, John Torpey (2006) argumenta que no es mera coincidencia que su proliferación se produzca en un contexto de colapso del socialismo y devaluación de los proyectos políticos explícitamente orientados hacia el futuro. Para el autor, este nuevo sentido de la "víctima" se relaciona con el surgimiento de la idea de los derechos humanos - con la consiguiente aparición de subgrupos e individuos como sujetos de derecho internacional - y con la "judicialización” de la política de manera general. Lo que está en marcha, para él, es una tendencia cultural a la recuperación de la víctima, a expensas del heroísmo, que nutre el resentimiento en lugar de valorar la adversidad y superación; en definitiva, un nuevo tema cultural relacionado con la resemantización del concepto colectivo de la víctima.

Entre las consecuencias negativas de esta resemantización, surge la extinción de la capacidad de agencia de los actores sociales y la responsabilidad individual por los fracasos que comparten. Tal individualización termina desconectando a la "víctima" de su contexto social, sin tener en cuenta que la historia de su vida se construye colectivamente y que su decisión de migración y desplazamiento tiene relación con contextos familiares o afectivos (Dias y Sprandel 2011).

En el campo de las migraciones internacionales, la transposición de la noción de "víctima criminal" a la de "víctima humanitaria" cuenta con diferentes implicaciones y tensiones. La celebración de una víctima genérica - carente incluso de historia - elimina la autonomía de los individuos, convirtiéndolos en seres inertes y sin agencia.

El concepto de "víctima" puede, por lo tanto, convertirse en una trampa cuando las políticas contra la trata de personas y tráfico ilícito de migrantes destacan el crimen organizado y la delincuencia. Esto legitima el régimen de deportación y eclipsa la cuestión más amplia de los derechos de las personas que emigran y trabajan en actividades diversas (Mahdavi 2011).

\section{ESPACIOS DE REGULACIÓN, CRIMINALIZACIÓN Y CONTROL:}

\section{LA PROTESTA DE REFUGIADOS EN VIENA}

En noviembre de 2012, más de un centenar de solicitantes de asilo y activistas caminaron del campo de refugiados Lager Traiskirchen, ubicado a 20 kilómetros al sur de Viena, hacia el centro de la ciudad. La mayoría de ellos procedían de la frontera entre Pakistán y Afganistán y protestaban contra las condiciones de vida en ese campo. Exigían el acceso al mercado de trabajo, la libertad de movimiento, además de una mayor velocidad en la adjudicación de las solicitudes de asilo. La protesta despertó el interés de los medios de comunicación y la opinión pública, ya que, contrariamente a la previsión de regreso a Traiskirchen, las personas decidieron acampar en el parque Sigmund Freud, uno de los principales puntos turísticos de la capital austriaca. 
La permanencia de las tiendas de los refugiados en el parque era un acontecimiento arriesgado, así como la consiguiente ocupación de la iglesia Votivkirche por alrededor de 90 personas en diciembre de ese año. La policía respondió retirando las tiendas del parque. Esto intensificó el conflicto y cerca de 50 refugiados empezaron una huelga de hambre que duró cerca de un mes dentro de la iglesia. La participación en las negociaciones del presidente Heinz Fischer y del cardenal Christoph Schönborn, máximo líder de la Iglesia Católica en Austria, muestra el grado de importancia que la protesta adquirió.

Varias iniciativas para cerrar la protesta fueron llevadas a cabo y los refugiados incluso recibieron una oferta para ser acomodados en otro centro de recepción y una propuesta no oficial de una visa humanitaria por un período de un año para interrumpir el movimiento. Después de más de tres meses en la Votivkirche, los refugiados hicieron nuevas negociaciones, salieron de la iglesia y se fueron a vivir al monasterio Serviten, gestionado por Cáritas. Gracias a la influencia del cardenal Schönborn, Cáritas también se comprometió a volver a prestar la asistencia básica que había sido interrumpida por considerar que los refugiados habían "desertado" de Traiskirchen.

Mientras tanto, se conocieron varios resultados de las solicitudes de asilo enviadas por miembros del movimiento. Veintisiete de las 60 solicitudes fueron rechazadas y no se aprobó ninguna solicitud de los refugiados registrados en el monasterio. Los que no han recibido una respuesta negativa siguieron a la espera de un juicio de sus peticiones.

Durante el este período, las movilizaciones de los refugiados permanecieron constantes. Marchas, actos públicos, exposiciones de arte y varias otras iniciativas ganaron visibilidad. Además, el movimiento ganó apoyo de diferentes sectores de la sociedad austriaca, como la Academia de Bellas Artes de Viena, personalidades y políticos del Partido Verde, así como numerosas organizaciones de derechos humanos que operan en la capital austriaca.

Sin embargo, en julio, 20 solicitantes que habían tenido sus decisiones de asilo denegadas recebieron la gelinderes Mittel, una carta pidiendo a la persona que vaya a la policía en los días siguientes. Ocho de estos individuos de origen paquistaní que acudieron a la policía fueron inmediatamente arrestados y deportados a Pakistán. Tampoco se consideró la posición del cardenal en la búsqueda de soluciones alternativas. Los otros refugiados, a su vez, se negaron a ir a la policía, y el temor a la deportación aumentó.

El período en que ocurrieran estas deportaciones coincidió con el momento de las elecciones en Austria. Varios activistas y periodistas relacionaban esta reacción desproporcionada de las autoridades a los intereses de la campaña. El partido FPO, de extrema derecha, utilizó el tema de los refugiados para despertar sentimientos anti-inmigrantes en el país. El Partido Popular, que estaba en el poder, utilizó la situación para defender el rigor 


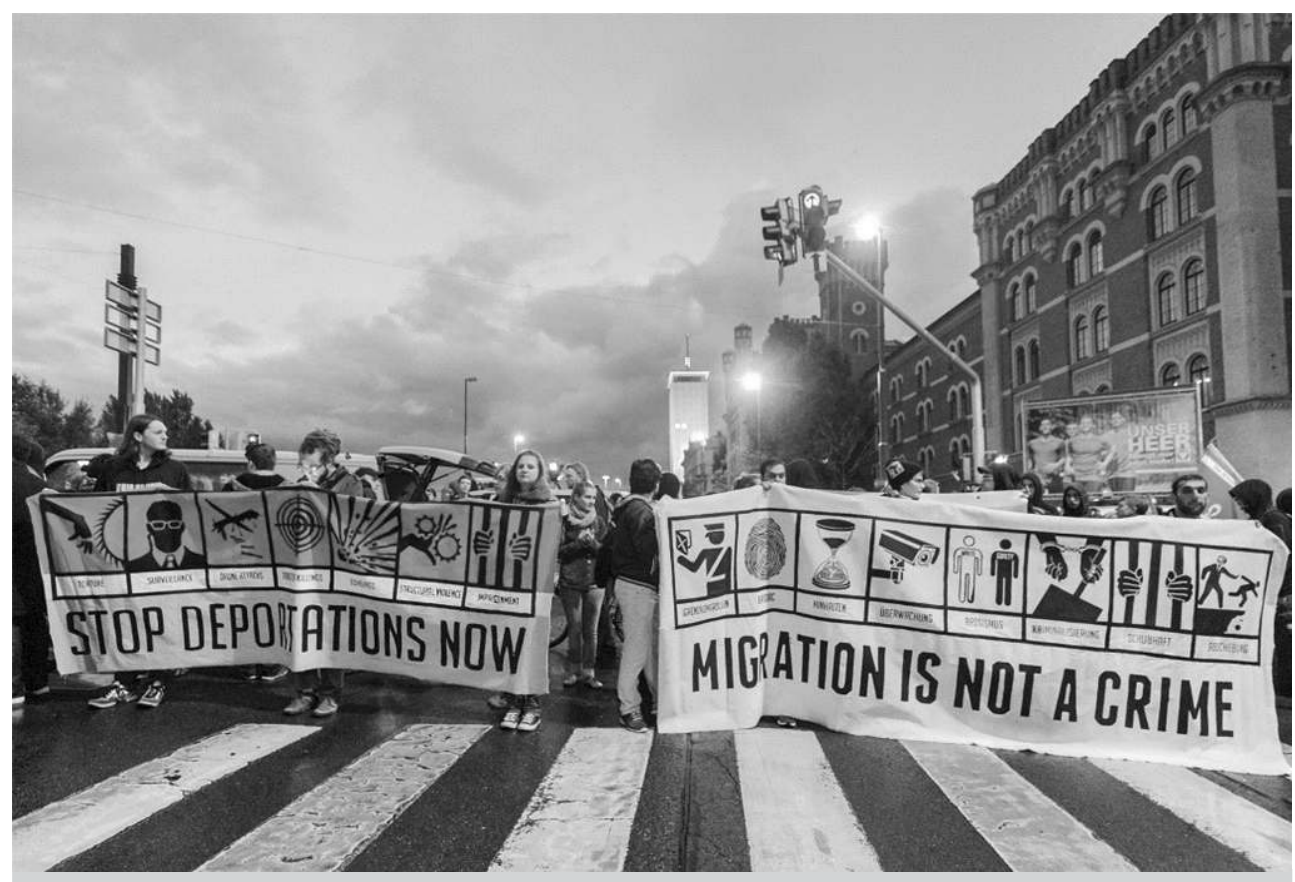

Figura 1 - Protesta en solidaridad con los refugiados, Viena, 20 de septiembre de 2013.

de sus operaciones. Ya algunos miembros del Partido Verde y organizaciones de derechos humanos defendieron a los inmigrantes, pidiendo el cese de las deportaciones.

A pesar de las protestas y campañas de solidaridad, la deportación de aquellas ocho personas a Pakistán conmocionó a los miembros del movimiento. Además, la persecución de sus miembros se dio a través de la criminalización. Desde la ocupación de la iglesia, se realizaron escuchas telefónicas en los celulares de los refugiados y activistas y el intento de criminalizar el movimiento ganó materialidad el 30 de julio de 2013, cuando seis refugiados (cuatro de ellos estaban en el monasterio) fueron detenidos y acusados de ser parte de una banda de contrabandistas de personas.

Los ocho acusados eran paquistaníes. Dos de ellos, con 18 y 19 años, fueron puestos en libertad de manera más inmediata por falta de pruebas de vinculación con cualquier actividad criminal. Los otros seis fueron detenidos, acusados de facilitar la inmigración ilegal y el tráfico ilícito de migrantes. La "evidencia" de las autoridades para los delitos vinculaba estas personas a una supuesta banda que introdujo extranjeros a Austria desde los Balcanes y la frontera con Hungría.

La ministra del Interior de Austria, Johanna Mikl-Leitner, no hizo ningún esfuerzo para diferenciar los crímenes cometidos por los individuos. En 
una entrevista para un noticiero de televisión, informó que la banda movía alrededor de "10 millones de euros" al año. Sus afirmaciones fueron negadas por periodistas, políticos y abogados, y ella necesitó hacer una advertencia diciendo que había hablado en general, y que los 10 millones de euros no solo vinieron de esa organización delictiva.

Según el periodista Florian Klenk, las alegaciones contenidas en los registros de la Fiscalía sobre las prácticas criminales que involucraban a los refugiados de Votivkirche eran "inofensivas" en comparación con las declaraciones de la ministra. En las alegaciones había acusaciones que incluían la ayuda dada a personas procedentes de Budapest o Italia desde la estación de tren, el suministro de alimentos para algunos de ellos, y los préstamos que oscilaban entre $30 €$ y $200 €{ }^{8}$

Las protestas que siguieron denunciaron la arbitrariedad de las medidas adoptadas y el proceso de criminalización del movimiento de refugiados. En conversaciones y reuniones a las que asistí durante el estudio de campo, las personas comprometidas con el movimiento mostraron su preocupación e indignación por el curso tomado por el movimiento. Criticaron su criminalización (principalmente debido a las acusaciones relacionadas con el crimen de tráfico de migrantes); el carácter discriminatorio de las decisiones sobre las solicitudes de asilo, en particular de aquellos más abiertamente involucrados en las protestas; el peligro de regreso para las regiones de las que provenía la mayoría en Pakistán (Khyber-Pakthunkhwa, Swat Valley); la falta de transparencia y la decodificación de documentos y procedimientos realizados en el proceso de solicitud de asilo; y la traición de las autoridades austriacas, que habían dado lugar a la deportación de parientes y conocidos.

Cabe destacar que ninguno de los acusados llegó a ser condenado. En marzo de 2014, el juez a cargo del caso dio la libertad a todos los involucrados, advirtiendo que las acusaciones eran demasiado vagas, que no había pruebas concretas sobre posibles crímenes cometidos y que las traducciones e intercepciones telefónicas realizadas por la policía fueron parcialmente engañosas.

A pesar del fracaso de las organizaciones internacionales especializadas en el tema migratorio en Viena para denunciar el uso pernicioso de las leyes contra la trata/tráfico en la criminalización de los movimientos de refugiados - con la honrosa excepción del documento "Report to the United Nations: deportation of protestors against inhumane conditions in refugee camps and asylum procedures in Austria" (Dr. Michael Platzer, 08/08/2013) -, el movimiento de los refugiados ha puesto de relieve las contradicciones del aparato

8 Ver entrevista publicada en < http://fm4.orf.at/stories/1722737/ > (última consulta en octubre de 2017). 


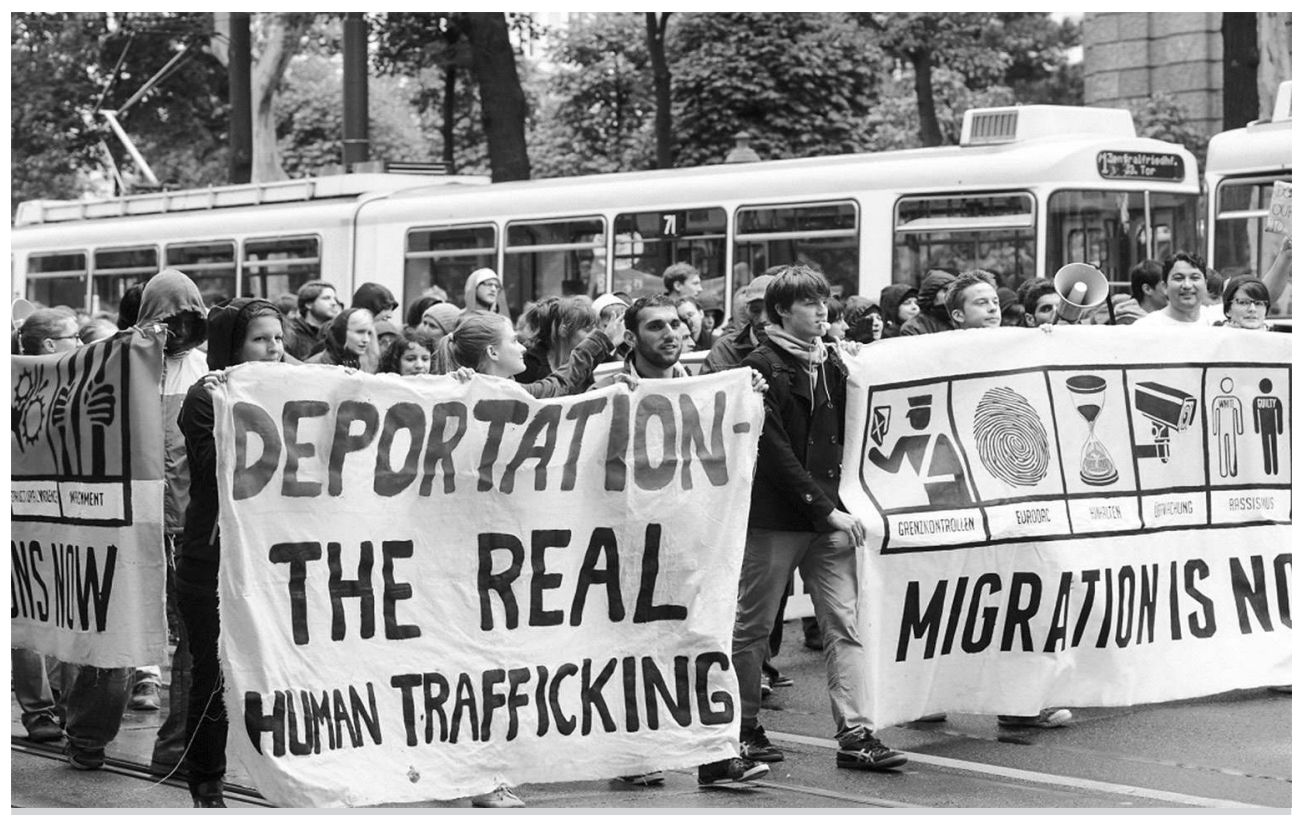

Figura 2 - Protesta en solidaridad con los refugiados, Viena, 20 de septiembre de 2013.

discursivo que combina el humanitarismo, la seguridad y la criminalización de los inmigrantes. ${ }^{9}$

El término "deportación", no utilizado por las autoridades, pero reiterado por los líderes del movimiento, otorga al Estado la responsabilidad de esta "verdadera forma de la trata de personas", y da señales sobre la arbitrariedad de las autoridades en el tratamiento de los solicitantes de asilo.

Los refugiados de Votivkirche están lejos de ser las víctimas que predica la escena humanitaria. De todos modos, la categoría fue lo que quedó para oponerse a la reacción desproporcionada del Estado austríaco contra el movimiento social. En el documento de apoyo firmado por el Dr. Platzer, por ejemplo, la ocupación de Votivkirche fue narrada como una necesidad del grupo para "escapar del frío y llamar la atención de las autoridades sobre o en relación

9 Al reflexionar en mi tesis doctoral (Dias 2014a: 150), destaqué como este fracaso sólo confirma la distancia entre el mundo de los migrantes y el mundo de los funcionarios internacionales que se ocupan del tema migratorio. Al menos cinco organizaciones internacionales con sede en Viena tienen departamentos o representaciones específicas sobre el tema de la trata de personas/contrabando de migrantes: la Oficina de las Naciones Unidas contra la Droga y el Delito (UNODC), la Organización para la Seguridad y la Cooperación en Europa (OSCE), la Organización Internacional para las Migraciones (OIM), la Agencia de los Derechos Fundamentales de la Unión Europea (FRA) y el Centro Internacional para el Desarrollo de Políticas Migratorias (ICMPD). Ninguno de ellos tenía cualquier posición con respecto al uso de las leyes contra el contrabando de migrantes para la criminalización del movimiento de refugiados de Viena. 
a las condiciones inhumanas en el campo". Es significativo que un movimiento con tanta legitimidad tenga necesidad, aunque contextualmente, de recurrir a la categoría de víctima para defenderse de las acciones arbitrarias del Estado austriaco.

De cualquier forma, la existencia del movimiento de Votivkirche pone al descubierto como las normas europeas e internacionales sobre la trata de personas/tráfico ilícito de migrantes engendran la criminalización como forma de control y regulación de la movilidad humana. Las leyes en Austria no se diferencian de las adoptadas por otros países europeos y siguen las directrices generales de las directivas europeas y el derecho internacional. En este caso, vimos cómo dieron margen y respaldo a la criminalización de los miembros del movimiento de Votivkirche.

El uso de estas políticas para criminalizar el movimiento de las personas que no están relacionadas con "bandas" u "organizaciones criminales" es, en este sentido, más una evidencia etnográfica que apunta a la nocividad de cierto discurso contra la trata/el tráfico en diferentes contextos regionales y que ha sido denunciado por la literatura especializada y autores que se dedican al tema.

En un contexto en que las nociones de ciudadanía y derechos dan lugar de forma cada vez más frecuente a la dicotomía víctima/criminal, varios sujetos han sido atrapados en los extremos de un tipo de clasificación peligrosa y sin matices. Es en este sentido que las medidas para combatir la trata de personas y el tráfico ilícito de migrantes en diferentes contextos contemporáneos han mostrado sus límites, rehenes de las prácticas de control y discriminación propagada por los Estados nacionales. 


\section{BIBLIOGRAFÍA}

ACNUDH - Oficina del Alto Comisionado de las Naciones Unidas para los Derechos Humanos, 2013, Declaración y Programa de Acción de Viena, Aprobados por la Conferencia Mundial de Derechos Humanos el 25 de junio de 1993, Oficina del Alto Comisionado de las Naciones Unidas para los Derechos Humanos, disponible en < http://www.ohchr. org/Documents/Events/OHCHR20/VDPA_booklet_Spanish.pdf > (última consulta en octubre 2017).

AGAMBEN, Giorgio, 2004, Estado de Exceção. Rio de Janeiro, Boitempo.

AGIER, Michel, 2006, "Refugiados diante da nova ordem mundial", Tempo Social, 18 (2): 197-215.

AUGUSTIN, Laura Maria, 2005, "La industria del sexo, los migrantes y la familia europea", Cadernos Pagu, 25: 107-128.

AUGUSTIN, Laura Maria, 2007, Sex at the Margins: Migration, Labour Markets and the Rescue Industry. Londres, Zed Books.

BERNSTEIN, Elizabeth, 2012, "Carceral politics as gender justice? The 'traffic in women' and neoliberal circuits of crime, sex, and rights”, Theory and Society, 41 (3): 233-259.

CASTILHO, Ela Wiecko V. de, 2013, "Problematizando o conceito de vulnerabilidade para o tráfico internacional de pessoas", en Tráfico de Pessoas: Uma Abordagem para os Direitos Humanos. Brasilia, Secretaria Nacional de Justiça - Ministério da Justiça, 133-153.

CLAVIJO, Janneth, y Maria José MAGLIANO, 2013, "La OIM como 'trafficking solver' para la región sudamericana: sentidos de las nuevas estrategias de control migratório”, en Gabriela Kasarik (comp.), Migraciones Internacionales: Reflexiones y Estudios sobre la Movilidad Territorial Contemporánea. Buenos Aires, Fundación Ciccus, 129-148.

DIAS, Guilherme Mansur, 2014a, Migração e Crime: Desconstrução das Políticas de Segurança e Tráfico de Pessoas. Campinas, Universidade Estadual de Campinas, tesis de doctorado en Antropología Social.

DIAS, Guilherme Mansur, 2014b, "Migração, segurança e governabilidade migratória: o papel dos organismos internacionais”, Crítica y Emancipación: Revista Latinoamericana de Ciencias Sociales, 11: 557-580.

DIAS, Guilherme Mansur, y Marcia Anita SPRANDEL, 201 1, "Reflexões sobre políticas para migrações e tráfico de pessoas no Brasil”, Revista Interdisciplinar da Mobilidade Humana (REMHU), 37: 59-77.

DOEZEMA, Jo, 2005, “Now you see her, now you don't: sex workers at the UN Trafficking Protocol negotiation”, Social \& Legal Studies, 14 (1): 61-89.

DOEZEMA, Jo, 2010, Sex Slaves and Discourse Masters: The Construction of Trafficking. Londres, Zed Books.

FASSIN, Didier, 2001, "Quand le corps fait la loi: la raison humanitaire dans les procédures de régularisation des étrangers", Sciences Sociales et Santé, 19 (4): 5-33.

FASSIN, Didier, 2006, "Souffrir par le social, gouverner par l'écoute: une configuration sémantique de l'action publique", Politix, 19 (73): 37-157.

KEMPADOO, Kamala, 2005, "Mudando o debate sobre o tráfico de mulheres", Cadernos Pagu, 25: 55-78.

KEMPADOO, Kamala, 2016, "Revitalizing imperialism: contemporary campaigns against sex trafficking and modern slavery”, Cadernos Pagu, 47: el 6478.

LEE, Maggy, 201 1, Trafficking and Global Crime Control. Londres, Routledge. 
MAHDAVI, Pardis, 2011, Gridlock: Labor, Migration and Human Trafficking in Dubai. Standford, CA, Stanford University Press.

OLÍVAR, José Miguel, 2016, "Exploring traffic and exploitation on the Brazilian international border in the Amazon", Social and Economic Studies, 65 (4): 57-86.

PISCITELLI, Adriana, 2008, "Entre as máfias e a ajuda: a construção de conhecimento sobre tráfico de pessoas", Cadernos Pagu, 31: 29-63.

PISCITELLI, Adriana, 2011 , "Procurando vítimas do tráfico de pessoas: brasileiras na indústria do sexo na Espanha", Revista Interdisciplinar da Mobilidade Humana (REMHU), 37: $11-26$.

PISCITELLI, Adriana, y Laura LOWENKRON, 2015, "Categorias em movimento: a gestão de vítimas do tráfico de pessoas na Espanha e no Brasil”, Ciência e Cultura, 67 (2): 35-39.

SILVA, Ana Paula da, y Thaddeus Gregory BLANCHETTE, 2010, "Mulheres vulneráveis e meninas más", en I Prêmio Libertas: Enfrentamento ao Tráfico de Pessoas. Brasilia, Secretaria Nacional de Justiça - Ministério da Justiça, 147-188.

SIMON, Jonathan, 2007, Governing through Crime: How the War on Crime Transformed American Democracy and Created a Culture of Fear. Oxford, Oxford University Press.

SPRANDel, Marcia Anita, 2004, A Pobreza no Paraíso Tropical. Rio de Janeiro, Relume Dumará.

SPRANDEL, Marcia Anita, y Guilherme Mansur DIAS, 2010, "A temática do tráfico de pessoas no contexto brasileiro", Revista Interdisciplinar da Mobilidade Humana (REMHU), 35: 155-170.

TEIXEIRA, Flávia, 2008, "L'Italia dei Divieti: Entre o sonho de ser européia e o babado da prostituição”, Cadernos Pagu, 31: 275-308.

TICKTIN, Miriam, 2008, "Sexual violence as the language of border control: where French feminist and anti-immigrant rhetoric meet", Signs: Journal of Women in Culture and Society, 33 (4): 863-889.

TORPEY, John, 2006, Making Whole What Has Been Smashed: On Reparation Politics. Cambridge, MA, Harvard University Press.

UNODC - United Nations on Drugs and Crime, 2004, Convención de las Naciones Unidas contra la Delincuencia Organizada Transnacional y Sus Protocolos. Nueva York, Naciones Unidas, disponible en < https://www.unodc.org/documents/treaties/UNTOC/Publications/ TOC\%20Convention/TOCebook-s.pdf > (última consulta en octobre 2017).

VELASCO, Soledad, 2015, "Desmontando ilusiones: el tráfico de migrantes irregularizados' más allá del crimen, el sentido común y los ilegalismos”, apresentación en la mesa redonda "Políticas Públicas en Torno a la Trata de Personas y el Tráfico de Migrantes: Desafíos para Su Formulación y Implementación”, Quito, FLACSO, 28 de mayo.

WONG, Diana, 2005, "The rumor of trafficking: border controls, illegal migration, and the sovereignty of the nation-state", en W. Schendel y I. Abraham (comps.), Illicit Flows and Criminal Things: States, Borders, and the Other Side of Globalization. Bloomington, Indiana University Press, 69-100. 